\title{
La gestion d'une bibliothèque parlementaire ou législative : un survol
}

An Overview of the Management of a Parliamentary Library

La administración de una biblioteca parlamentaria 0

legislativa: panorama general

\section{Richard Paré}

Volume 47, numéro 4, octobre-décembre 2001

Les bibliothèques parlementaires

URI : https://id.erudit.org/iderudit/1030372ar

DOI : https://doi.org/10.7202/1030372ar

Aller au sommaire du numéro

\section{Éditeur(s)}

Association pour l'avancement des sciences et des techniques de la

documentation (ASTED)

\section{ISSN}

0315-2340 (imprimé)

2291-8949 (numérique)

Découvrir la revue

\section{Citer cet article}

Paré, R. (2001). La gestion d'une bibliothèque parlementaire ou législative : un survol. Documentation et bibliothèques, 47(4), 133-137.

https://doi.org/10.7202/1030372ar

\section{Résumé de l'article}

L'auteur passe en revue les différentes fonctions de gestion reliées à l'administration d'une bibliothèque parlementaire ou législative, y compris la gestion des collections, la gestion des ressources humaines, des ressources financières et la gestion des nouvelles technologies. Il conclut avec quelques commentaires sur la structure organisationnelle.
Tous droits réservés (C) Association pour l'avancement des sciences et des techniques de la documentation (ASTED), 2001
Ce document est protégé par la loi sur le droit d'auteur. L'utilisation des services d'Érudit (y compris la reproduction) est assujettie à sa politique d'utilisation que vous pouvez consulter en ligne.

https://apropos.erudit.org/fr/usagers/politique-dutilisation/ 


\title{
La gestion d'une bibliothèque parlementaire ou législative: un survol
}

\author{
Richard Paré \\ Bibliothèque du Parlement, Ottawa
}

L'auteur passe en revue les différentes fonctions de gestion reliées à l'administration d'une bibliothèque parlementaire ou législative, $y$ compris la gestion des collections, la gestion des ressources humaines, des ressources financières et la gestion des nouvelles technologies. II conclut avec quelques commentaires sur la structure organisationnelle.

\begin{abstract}
An Overview of the Management of a Parliamentary Library The author summarises the various management functions associated with a parliamentary or legislative library, such as collections management, human resources management, financial management and the management of technologies. He concludes with a couple of remarks about the organisational structure.
\end{abstract}

\begin{abstract}
La administración de una biblioteca parlamentaria o legislativa : panorama general

El autor pasa revista a las diferentes funciones administrativas comprendidas en la gestión global de una biblioteca parlamentaria o legislativa, entre las que se cuentan la administración de las colecciones, de los recursos humanos, de los recursos financieros, y de las nuevas tecnologías, para concluir su artículo ofreciendo algunos comentarios sobre la estructura organizativa.
\end{abstract}

La notion de gestion d'une bibliothèque parlementaire ou législative, semblable à celle de la gestion d'un service avec toutes les particularités qui s'y rattachent, se définit comme étant le développement, la mise en œuvre et le contrôle de toutes les activités liées à la prestation d'un service de bibliothèque aux législateurs et au personnel d'un Parlement ou d'une Assemblée législative. II peut arriver, dans certains cas, que d'autres services soient ajoutés aux responsabilités traditionnelles de la bibliothèque parlementaire ou législative.

C'est le cas, par exemple, aux ÉtatsUnis où le Congressional Research Service fournit au Sénat et à la Chambre des représentants, en plus des services de bibliothèque, des services d'analyse et de recherche. On retrouve des situations similaires à la Bibliothèque des Parlements britannique, canadien et australien et des Assemblées législatives de l'Ontario et du Québec entre autres, où l'on offre des services de bibliothèque et de recherche aux législateurs.

Certaines bibliothèques parlementaires ou législatives se voient attribuer d'autres responsabilités. C'est le cas; par exemple, de la Bibliothèque de la Chambre des communes de Westminster qui gère un Service de renseignements destiné au public. On retrouve une situation équivalente à la Bibliothèque du Parlement canadien, qui compte dans son mandat la responsabilité de fournir des services, des programmes éducatifs et de l'information sur le Parlement au grand public. Dans ce contexte, la Bibliothèque répond aux demandes de renseignements sur le Parlement, assure un service de guides pour les visites du Parlement, coordonne au nom du Sénat et de la Chambre des communes un forum annuel pour les enseignants sur le fonctionnement du Parlement et, enfin, gère une boutique "souvenirs-cadeaux » à l'intention des visiteurs et des parlementaires.

\section{Clientèles}

Comme bon nombre de bibliothèques parlementaires et législatives fournissent des services d'information, de documentation et d'analyse, il importe donc d'examiner de façon générale les paramètres qui déterminent les particularités d'une clientèle parlementaire ou législative.

Dans la plupart des grandes démocraties du monde, les parlementaires sont élus et représentent leurs commettants, le plus souvent dans le cadre de partis politiques reconnus selon les systèmes établis dans chaque pays. II arrive aussi que des parlementaires soient nommés et non élus. C'est le cas, par exemple, pour le Sénat au Canada et à la Chambre des Lords en Angleterre.

Les parlementaires élus le sont habituellement pour des mandats variant de quatre à cinq ans. La durée de ces mandats peut être plus courte selon les conditions politiques qui prévalent dans chaque Parlement et/ou législature. Par exemple, si les membres des partis d'opposition peuvent, au total des voix, défaire les membres du parti politique au pouvoir, il y a de fortes chances que ce Parlement ou cette législature dure moins de quatre ans.

Ceci signifie que la clientèle des bibliothèques parlementaires ou législatives se renouvelle en partie, plus ou moins, à tous les quatre ans. II faut ajouter à ce constat le personnel au service des parlementaires qui suit à peu près le même scénario. II arrive souvent que les services de la bibliothèque parlementaire ou législative soient aussi offerts à d'autres groupes de clients tels les fonctionnaires du gouvernement et même au public.

La connaissance de sa clientèle constitue évidemment un atout majeur pour une entreprise de service telle une bibliothèque parlementaire ou législative. Comme il s'agit d'une clientèle ciblée, il est donc très important d'établir rapidement, surtout au début de chaque législature, le profil d'intérêt des divers partis politiques repré- 
sentés ainsi que celui de chaque député ou sénateur. Les facteurs à considérer sont les orientations politiques décrites dans les programmes des parties et les antécédents dans la carrière antérieure du parlementaire, sa participation à un ou des comités spécifiques et les principaux intérêts de la région qu'il représente.

À partir d'un tel profil, il sera alors possible d'offrir une information encore plus pertinente reliée aux besoins identifiés et de développer les outils spécialisés nécessaires qui permettront de trouver rapidement et facilement l'information recherchée.

\section{Gestion des collections}

Les bibliothèques parlementaires et législatives sont en principe des bibliothèques spécialisées puisqu'elles desservent une ou des clientèles ciblées et qu'elles fournissent souvent des services particuliers à leurs clients. Par contre, l'intérêt des parlementaires est très diversifié et couvre d'innombrables champs de connaissances, ce qui se traduit par un développement de collections qui touche à tous les domaines du savoir. On peut donc avancer, sans crainte de se tromper, qu'une bibliothèque parlementaire ou législative possède une vaste collection touchant à tous les domaines. On pourrait, à ce titre, la définir comme une bibliothèque spécialisée en généralités.

La bibliothèque devra compter d'abord sur une excellente collection d'ouvrages de référence et donner l'accès à une collection de bases de données qui permettra de répondre à tout genre de questions dites factuelles afin de répondre aux intérêts diversifiés de sa clientèle spécifique.

De plus, toute bibliothèque parlementaire ou législative aura intérêt à développer des collections importantes dans les domaines des affaires politiques et parlementaires entourant le pays, la province ou la région géographique où elle est située, des affaires gouvernementales, des sciences économiques et sociales, du droit et de la législation, de la procédure parlementaire, des relations nationales et internationales et, enfin, de l'histoire du pays, de la province ou de l'État concerné.

La sélection des nouvelles acquisitions peut se faire selon différents processus ou modèles. II est tout d'abord assuré qu'une suggestion d'acquisition faite par un parlementaire aura la priorité dans la «chaîne » de sélection. À moins qu'une telle acquisition soit tout à fait injustifiable ou trop coûteuse en fonction des budgets alloués, il est peu probable qu'elle soit rejetée.

Habituellement, la sélection des nouvelles acquisitions se fait selon une politique et certaines lignes directrices préétablies. Dans l'application de cette politique, les méthodes ou modèles diffèrent d'un endroit à l'autre. D'abord, disons que le personnel professionnel de la bibliothèque qui doit répondre aux demandes des parlementaires et des législateurs est souvent bien placé pour faire des recommandations quant aux nouvelles acquisitions.

Les deux modèles qui se trouvent dans la majorité des bibliothèques les plus importantes peuvent se décrire à peu près comme suit. La bibliothèque où la responsabilité de la sélection des nouvelles acquisitions est déléguée à un ou à quelques membres du personnel spécialisé dans le domaine. Habituellement, ces bibliothécaires sont aussi impliqués de très près dans les activités d'émondage ou de désherbage et de conservation des collections. Ils participent généralement à la préparation de la politique de développement des collections et voient à l'application de toutes ces facettes.

Un autre modèle fait partager la sélection des nouveaux ouvrages par une équipe ou un ensemble de membres du personnel, ce qui permet une couverture plus vaste des divers champs d'intérêt déjà identifiés dans la politique de développement des collections.

À la Bibliothèque de l'Assemblée nationale du Québec, on a implanté, en 1978, un modèle basé sur les grandes classes de la classification décimale Dewey, qui invitait le personnel (bibliothécaires, attachés de recherche, bibliotechniciens) à participer à la sélection des nouveaux livres et documents. Le modèle fonctionnait à peu près comme ceci: chaque participant choisissait, selon ses connaissances et son expertise professionnelle, un secteur ou domaine principal du développement des collections comme, par exemple, les sciences et affaires politiques, l'économie, les affaires sociales, les sciences et la technologie, l'histoire québécoise et canadienne, la gestion et les affaires publiques. Chacun pouvait également ajouter un secteur secondaire selon ses goûts personnels, comme les arts, les sports, la philosophie, la littérature. Chaque participant pouvait aussi suggérer des ouvrages de référence reliés aux sujets d'intérêt choisis et le coordonnateur du programme assurait le choix des ouvrages généraux.

L'avantage de ce modèle est qu'il permet une vaste couverture de tous les domaines du savoir par des équipes intéressées par les sujets choisis. Il a aussi l'avantage de mettre en valeur les connaissances et l'expertise de plusieurs membres du personnel dont la bibliothèque n'aurait pu profiter autrement. II reste que peu importe le modèle que l'on favorise pour la sélection de la documentation, le responsable ou le coordonnateur de cette activité veille au bon équilibre entre les différents domaines; il s'assure surtout que l'on pourra répondre efficacement à toute demande venant du parlementaire, du législateur ou de son personnel.

Après la sélection, il faut voir à la gestion efficace du processus d'acquisition, de catalogage et de classification, de la préparation matérielle et de la conservation des livres, périodiques et autres documents, et de la circulation.

L'arrivée des nouvelles technologies et l'utilisation accrue des ordinateurs depuis les trente dernières années environ ont permis d'automatiser les opérations d'acquisition, de catalogage et de circulation et ainsi assurer une gestion plus rapide et intégrée de cette chaîne documentaire et des collections de livres en format numérisé.

La gestion des collections nécessite de plus en plus un personnel qualifié dans le commerce du livre, des publications en série, des périodiques, des journaux et de l'information en format électronique. En plus d'effectuer des transactions dans le domaine de l'imprimé, le personnel responsable des acquisitions d'une bibliothèque doit maintenant être en mesure de négocier des contrats donnant accès à des bases de données bibliographiques ou documentaires et à des collections de livres en format numérisé.

Cette nouvelle tendance à la haússe $a$ amené les bibliothèques parlementaires et législatives à participer à des consortia de bibliothèques qui, en se regroupant, peuvent ainsi négocier des permis ou des licences d'utilisation à de meilleurs taux. Par exemple, la Bibliothèque du Parlement canadien participe au consortium des bibliothèques du gouvernement fédéral, ce 
qui lui permet de bénéficier de «prix de groupes» pour plusieurs services de bases de données et ainsi réaliser des économies substantielles.

La gestion des collections en format électronique constitue un défi de taille puisqu'elle soulève différentes questions autant sur le plan de l'évaluation et de la sélection des publications ou documents électroniques que sur le plan de la qualité même des documents en format numérisé. Une pratique courante consiste à faire une photocopie d'un document électronique dont on a décidé d'ajouter le lien de l'adresse électronique au catalogue de la bibliothèque. La copie imprimée est ajoutée aux collections ce qui assure la pérennité du document.

Plusieurs bibliothèques parlementaires et législatives ont hérité et/ou reçoivent en cadeaux, dons ou échanges, de nombreux volumes rares et historiques qui ont une valeur incommensurable. La gestion de ces collections spéciales nécessite une attention particulière autant sur le plan de la protection et de la conservation que sur. le plan de la mise en valeur.

\section{Gestion des ressources humaines}

Le personnel d'une bibliothèque parlementaire ou législative constitue la ressource principale permettant d'assurer un service d'excellence aux parlementaires et législateurs et, avec le développement de collections appropriées, il forme la synergie nécessaire pour atteindre cet objectif.

Durant la première demie du $X X^{e}$ siècle, on note en général que le personnel des bibliothèques parlementaires et législatives, bien que très dévoué, était peu qualifié en bibliothéconomie, et que souvent il développait son expertise «sur le tas». Cette situation a changé progressivement par la suite et les universités comptent parmi leurs diplômés plus de bibliothécaires et de spécialistes de l'information.

Avant les années 1950, on pouvait noter, à la Bibliothèque du Parlement canadien, l'emploi de plusieurs commis qui avaient été référés par des députés et des sénateurs. Il y a même eu quelques cas où la lignée se poursuivait de père en fils. Bien qu'elles existent encore dans certaines bibliothèques parlementaires et législatives, ces pratiques de favoritisme ont été largement remplacées durant la se- conde partie du $X X^{e}$ siècle par la mise en place de processus et de pratiques de sélection du personnel plus équitables.

Le processus de dotation prévoit habituellement un concours auquel sont soumis les candidats admissibles aux postes à combler. Ce concours, qui permet d'évaluer les connaissances et les compétences des candidats, assure un processus de sélection plus impartial pour l'ensemble des candidats. Comme les bibliothèques parlementaires et législatives sont des services qui œuvrent dans le giron des diverses instances politiques, il est normal que les membres du Parlement ou de la législature reçoivent des demandes d'appui de leurs commettants désireux de poser leur candidature pour un poste à la bibliothèque.

II faut donc réussir à mettre en place un mécanisme qui permet à la fois de satisfaire le parlementaire et le commettant. À la Bibliothèque du Parlement canadien, nous recevons toutes les candidatures qui nous sont soumises par les députés ou les sénateurs et nous les conservons en dossier pour une période de deux ans. Nous informons le parlementaire et le candidat que ce dernier sera invité au concours de sélection si la Bibliothèque dispose d'un poste pour lequel le candidat est qualifié. Ce processus, en usage depuis plusieurs années, a jusqu'à maintenant donné de bons résultats.

Une autre facette dont il faut tenir compte dans la gestion des ressources humaines des bibliothèques parlementaires et législatives est la diversité des groupes d'employés particulièrement significative et importante comparativement aux autres types de bibliothèques, tels les bibliothèques d'universités et de collèges ou les bibliothèques publiques. En effet, en plus d'y retrouver des bibliothécaires, des techniciens en documentation, du personnel de soutien, on y trouve des gestionnaires de bases de données, des techniciens en informatique, et s'il existe un service de recherche rattaché à la bibliothèque, des avocats, des économistes, des scientifiques, des politicologues, des démographes ou des statisticiens, etc. qui devront travailler ensemble.

Cette sifuation où l'on retrouve dans la même organisation des spécialistes avec des antécédents académiques différents génère un bassin de connaissances et d'expertises qui ne peut qu'être bénéfique au Parlement ou à la législature. Cela signifie cependant que le gestionnaire doit, à tout prix, amener cette équipe multidisciplinaire à travailler ensemble, ce qui représente un défi de taille compte tenu des connaissances diversifiées et des personnalités souvent différentes.

II appert, cependant, qu'un personnel professionnel et technique tout à fait dévoué au Parlement ou à la législature est le meilleur gage d'un service de qualité si bien que la direction de la bibliothèque doit déployer tous les efforts nécessaires afin de développer et de maintenir ce dévouement.

\section{Gestion des ressources financières}

La plupart des bibliothèques parlementaires et législatives ont la responsabilité des budgets de leur organisation. À cet effet, les responsables des bibliothèques doivent préparer des plans en tenant compte des coûts des opérations régulières et des nouveaux projets, s'il y a lieu. Les salaires des employés et l'achat des livres ainsi que d'autres publications (imprimées et électroniques) constituaient traditionnellement la majeure partie du budget de fonctionnement de la bibliothèque. Mais, aujourd'hui, le coût des équipements informatiques et des réseaux de communication doivent être dûment planifiés et inclus dans le processus budgétaire, car ils comptent pour une large part des dépenses régulières de la bibliothèque.

À la Bibliothèque du Parlement canadien, le budget principal des dépenses couvre l'année financière qui s'étend d'avril jusqu'en mars. II est préparé en consultation avec les représentants des deux institutions parlementaires et soumis aux présidents du Sénat et de la Chambre des communes pour analyse et approbation. II est ensuite entériné par le Conseil du Trésor.

La Bibliothèque du Parlement prépare à chaque année, au printemps, à l'instar des ministères et agences du gouvernement canadien, son Rapport sur les plans et priorités. Ce rapport regroupe les services de la Bibliothèque par fonction et activité en indiquant les projets et les dépenses prévus pour une période d'une année financière et inclut une projection sur trois ans. Après la période de ladite année financière, la Bibliothèque produit son 
Rapport sur le rendement qui fait état des fonctions et activités préétablies dans le Rapport sur les plans et priorités et indique les résultats obtenus à partir d'indicateurs de rendement prédéterminés.

Ces pratiques visant à établir les plans, à identifier les priorités et, par la suite, à s'assurer que les ressources financières attribuées à la Bibliothèque ont été dépensées à bon escient se retrouvent sous une forme ou une autre dans toutes les bibliothèques parlementaires de moyenne ou de grande envergure. Dans les petites bibliothèques parlementaires ou législatives, ce processus de planification et de rétroaction se retrouve le plus souvent à un autre niveau de la législature ou du Parlement en question.

\section{Gestion des nouvelles technologies}

Depuis une trentaine d'années environ, les bibliothèques parlementaires et législatives ont subi l'impact des nouvelles technologies et plusieurs de leurs activités en ont été considérablement transformées. La première transformation à faire son apparition a été le développement de catalogues automatisés assistés par ordinateur qui s'est concrétisé surtout dans les années 1970 et 1980 . Pour le gestionnaire, il fallait d'abord faire l'analyse des besoins afin de choisir le système le plus approprié.

Après l'analyse des besoins, la sélection du système et son implantation, il a fallu délaisser progressivement la gestion des catalogues sur fiches pour ne s'en tenir qu'à la gestion des fichiers ordinolingues. Il est à prévoir que le catalogue automatisé se transformera petit à petit en base de données plein texte et constituera éventuellement une des composantes majeures de la bibliothèque parlementaire numérique.

L'arrivée des catalogues ordinolingues dans les bibliothèques parlementaires et législatives a rapidement donné lieu au développement d'autres produits électroniques - surtout dès l'implantation de réseaux locaux (intranet) de communication - permettant un accès et un échange rapide d'information sur un campus ou une cité parlementaire dans un périmètre prédéfini et à une clientèle privilégiée. Par exemple, l'accessibilité de la presse électronique a donné lieu rapidement au développement de services électroniques de monitoring des nouvelles - domaine d'information dont les politiciens et leur personnel sont très friands. À la Bibliothèque du Parlement canadien, ce service d'information électronique, développé sous l'acronyme PARLMEDIA, est celui qui est le plus utilisé sur une base quantitative parmi tous les produits et services électroniques offerts par la Bibliothèque.

D'autres services similaires d'information électronique peuvent s'ajouter au réseau local comme l'accès à une tour de cédéroms permettant la consultation en direct et en tout temps d'une collection d'ouvrages de référence (sur cédéroms). L'utilisation de ces outils électroniques sur CD crée progressivement un impact sur la nature des questions adressées au service d'information et de référence.

À titre d'exemple, à la Bibliothèque du Parlement canadien, le nombre de questions d'information factuelle a diminué considérablement au cours des dernières années; cependant, le nombre de questions complexes a augmenté. Ceci s'explique par le fait que les clients ont directement accès, à partir de leur bureau, à de l'information leur permettant de trouver eux-mêmes les réponses aux questions factuelles. Dès qu'il devient plus ardu de trouver l'information qu'ils recherchent, ils transmettent leur demande au Service de référence de la Bibliothèque.

L'usage des réseaux locaux permet aussi le développement d'instruments de gestion électronique des requêtes adressées à la bibliothèque parlementaire ou législative. Ces outils permettent aux clients de soumettre électroniquement à partir de leur ordinateur des demandes d'information, de recherche, d'analyse et d'emprunt d'ouvrages. Ce type de service de demandes en direct, qui est disponible 24 heures par jour et 7 jours par semaine, est sécuritaire et confidentiel. Bon nombre des requêtes électroniques commandent une réponse en format électronique. Ceci permet au personnel de la Bibliothèque de créer une base de données avec les questions reçues et les réponses fournies, ce qui s'avère très utile pour répondre rapidement aux prochaines questions similaires.

Pour assurer l'efficacité au niveau de l'exploitation des nouvelles technologies, il importe de s'assurer que tous les groupes d'usagers d'un réseau local d'une cité parlementaire ou législative utilisent les mêmes types d'équipements et les mêmes logiciels d'opérations, ce qui permet le ren- dement optimal du réseau à de meilleurs coûts à moyen et long termes.

La gestion des technologies de l'information dans les bibliothèques parlementaires et législatives présente un défi de taille. Les parlementaires et les législateurs, pour vaquer aux affaires de leur bureau et communiquer avec leurs commettants dans leur circonscription, s'attendent à ce que leur bibliothèque leur fournisse des services d'information et de recherche électroniques appropriés et rapides afin de les appuyer dans leurs fonctions de législateur et de représentation auprès de leurs commettants.

\section{Structure et organisation}

La structure organisationnelle des bibliothèques parlementaires et législatives peut varier d'un endroit à l'autre, mais comporte des composantes semblables à celles des autres types de bibliothèques.

Le chef de la bibliothèque relève en général du greffier du Parlement ou de l'Assemblée législative, ce qui est le cas dans la plupart des Parlements unicaméraux et Assemblées législatives provinciales. La situation peut être différente dans des Parlements bicaméraux où les parlementaires des deux Chambres se partagent les services d'une seule bibliothèque. C'est le cas, par exemple, au Parlement canadien où la Bibliothèque dessert le Sénat et la Chambre des communes et le bibliothécaire parlementaire relève des présidents des deux Chambres.

En plus des composantes traditionnelles que l'on retrouve dans la majorité des bibliothèques, plusieurs bibliothèques parlementaires et législatives offrent des services particuliers et différents. Nous avons déjà mentionné brièvement les services de recherche et d'analyse du Congressional Research Service aux États-Unis, de la Chambre des communes en Angleterre, du Parlement de Canberra, du Parlement du Canada et des Assemblées législatives de l'Ontario et du Québec. Nous avons également souligné les services d'information au public qui sont intégrés à la bibliothèque de la Chambre des communes de Westminster en Angleterre. II en va de même à la Bibliothèque du Parlement canadien où les programmes parlementaires destinés au public sont rattachés à la $\mathrm{Bi}$ bliothèque. 
À l'Assemblée nationale du Québec, citons à ce titre le service spécial de la reconstitution des débats où, depuis plus de vingt ans, on s'occupe à partir de la documentation de la presse et autres documents d'information publique de reconstituer les débats de l'Assemblée pour la période précédant la publication du Journal des débats.

Ce qui importe dans la structure organisationnelle, c'est de bien refléter tous les services définis dans le mandat et reliés à la mission; le mandat étant établi par l'autorité de qui relève la bibliothèque et la mission étant rédigée par les responsables de la bibliothèque qui doivent la faire approuver par les autorités compétentes.

À partir d'objectifs précis rattachés au mandat et à la mission de leur organisation, les gestionnaires de bibliothèques parlementaires ou législatives ont la responsabilité de planifier, de mettre en œuvre et de gérer le plus efficacement possible les activités nécessaires à la prestation de produits et services requis par les parlementaires et leur personnel.
En guise de conclusion à ce survol de la gestion d'une bibliothèque parlementaire ou législative, disons que les principaux acteurs qui forment la clientèle, soit les sénateurs et les députés, doivent participer à un grand nombre de fonctions et d'activités autant reliées à leur rôle de représentation auprès de leurs commettants qu'à celui de législateur. Par conséquent, ils font face au quotidien à un emploi du temps chargé et doivent donc pouvoir compter sur les meilleurs services de soutien possible, dont ceux de la bibliothèque parlementaire ou législative, de l'information, de la recherche et de l'analyse. La saine gestion et l'excellence des services offerts sont garantes de leur efficience et de leur efficacité. 